Marquette University

e-Publications@Marquette

Biological Sciences Faculty Research and

Publications

Biological Sciences, Department of

$8-2000$

\title{
Physiology of a Microgravity Environment Invited Review: Microgravity and Skeletal Muscle
}

Robert H. Fitts

Marquette University, robert.fitts@marquette.edu

Danny R. Riley

Medical College of Wisconsin

Jeffrey J. Widrick

Oregon State University

Follow this and additional works at: https://epublications.marquette.edu/bio_fac

Part of the Biology Commons

\section{Recommended Citation}

Fitts, Robert H.; Riley, Danny R.; and Widrick, Jeffrey J., "Physiology of a Microgravity Environment Invited Review: Microgravity and Skeletal Muscle" (2000). Biological Sciences Faculty Research and Publications. 593.

https://epublications.marquette.edu/bio_fac/593 
Marquette University

\section{e-Publications@Marquette}

\section{Biology Faculty Research and Publications/College of Arts and Sciences}

This paper is NOT THE PUBLISHED VERSION; but the author's final, peer-reviewed manuscript. The published version may be accessed by following the link in th citation below.

Journal of Applied Physiology, Vol. 89, No. 2 (August 2000): 823-839. DOI. This article is (C) American Physiological Society and permission has been granted for this version to appear in e-

Publications@Marquette. American Physiological Society does not grant permission for this article to be further copied/distributed or hosted elsewhere without the express permission from American Physiological Society.

\section{Physiology of a Microgravity Environment Invited Review: Microgravity and skeletal muscle}

Robert H. Fitts

Department of Biology, Marquette University, Milwaukee

Danny R. Riley

Department of Cellular Biology and Anatomy, Medical College of Wisconsin, Milwaukee, Wisconsin Jeffrey J. Widrick

Department of Exercise and Sport Sciences, Oregon State University, Corvallis, Oregon

\section{Abstract}

Spaceflight (SF) has been shown to cause skeletal muscle atrophy; a loss in force and power; and, in the first few weeks, a preferential atrophy of extensors over flexors. The atrophy primarily results from a reduced protein synthesis that is likely triggered by the removal of the antigravity load. Contractile proteins are lost out of proportion to other cellular proteins, and the actin thin filament is lost disproportionately to the myosin thick filament. The decline in contractile protein explains the 
decrease in force per cross-sectional area, whereas the thin-filament loss may explain the observed postflight increase in the maximal velocity of shortening in the type I and lla fiber types. Importantly, the microgravity-induced decline in peak power is partially offset by the increased fiber velocity. Muscle velocity is further increased by the microgravity-induced expression of fast-type myosin isozymes in slow fibers (hybrid I/II fibers) and by the increased expression of fast type II fiber types. SF increases the susceptibility of skeletal muscle to damage, with the actual damage elicited during postflight reloading. Evidence in rats indicates that SF increases fatigability and reduces the capacity for fat oxidation in skeletal muscles. Future studies will be required to establish the cellular and molecular mechanisms of the SF-induced muscle atrophy and functional loss and to develop effective exercise countermeasures.

Space exploration of the past 30 years, and particularly the Skylab, Spacelab, Cosmos, and Mir flights, have identified major biological changes with weightlessness in a variety of organ systems $(\underline{15}, \underline{21}, \underline{35})$. In the next few years, the International Space Station (ISS) will become operational. One of the primary objectives of ISS research will be to study and gain a clear understanding of microgravity-induced changes in biological processes with the ultimate goal of developing effective countermeasures such that exploration of Mars and points beyond can become a reality. One of the most affected systems is the neuromuscular system $(\underline{15}, \underline{21}, \underline{35})$. Weightlessness has been shown to cause atrophy, reduced functional capacity, and increased fatigue in limb skeletal muscles, with the greatest change observed in antigravity muscles such as the soleus (Sol) $(\underline{11}, \underline{73}, \underline{78}, \underline{99})$.

The purpose of this review is to identify the major effects of space travel on limb muscle structure and function, highlight those that present potential limitations to prolonged space travel and/or the crews' ability to readjust to $1 \mathrm{G}$, and discuss potential countermeasures. Special emphasis will be placed on how alterations in structure affect function and on publications that have addressed the cellular and molecular causes of the microgravity-induced muscle changes. The review will concentrate on spaceflight (SF) results and contrast and compare changes observed in rats and humans. Selected data from models of weightlessness [hindlimb unloading $(\mathrm{HU})$ in rats and bed rest in humans] will be discussed to support hypotheses for which insufficient space data exist. No attempt will be made to provide a complete review of the topic. For this, the reader is referred to a number of recent reviews in which the effects of SF on limb muscles in rodents $(\underline{21}, \underline{25}, \underline{71}, \underline{75})$ and humans $(\underline{15}, \underline{19}, \underline{21}, \underline{85})$ have been discussed in great detail.

\section{MICROGRAVITY AND LIMB MUSCLE MASS}

Skeletal muscle mass in rats .

Since the mid-1970s, it has been recognized that SF induces substantial atrophy of mammalian muscle, especially in muscles that play an antigravity, postural role in a $1-G$ environment. Early results from the Cosmos biosatellite program revealed rodent Sol muscle mass losses of $>30 \%$ after $20-22$ days in space (44). It is now known that this atrophic response occurs quite rapidly in microgravity, with reductions in rodent Sol mass of up to $37 \%$ observed after only 4-7 days of $S F(\underline{11}, \underline{20}, \underline{49}, \underline{58})$. In rats, it has consistently been observed that antigravity slow muscles such as the Sol and adductor longus (AL) atrophy more than primarily fast muscles and that extensors are more affected than flexors. For example, the slow type I fiber shows greater SF-induced atrophy than the fast type II fiber, and fibers 
from extensor muscles are more affected than those from flexors (Fig.1. . Edgerton and colleagues $(\underline{47}, 68)$ evaluated fiber size in Sol, medial gastrocnemius (MG), and tibialis anterior (TA) muscles after a 14-day SF (Cosmos 2044). Slow type I fibers from the ankle extensor muscles showed the greatest atrophy, with Sol fibers atrophying more than MG fibers (Fig. 1). Both slow and fast fibers of the TA showed a shift toward larger fibers postflight. Tischler et al. (194) demonstrated that the slow extensor muscles of young rats ( 26 days old) also show an increased susceptibility to SF-induced atrophy. The muscle weights of the Sol, plantaris, and gastrocnemius decreased by 38,24 , and $16 \%$, respectively, after a 5.4-day SF [Space Transport System (STS)-48], whereas the TA and extensor digitorum longus (EDL) muscles showed no change. Studies from this same flight showed that the muscle atrophy was associated with an increase in interstitial fluid volume (IFV) (41). The authors concluded that the loss of muscle mass and contractile protein was at least in part responsible for the increased IFV.

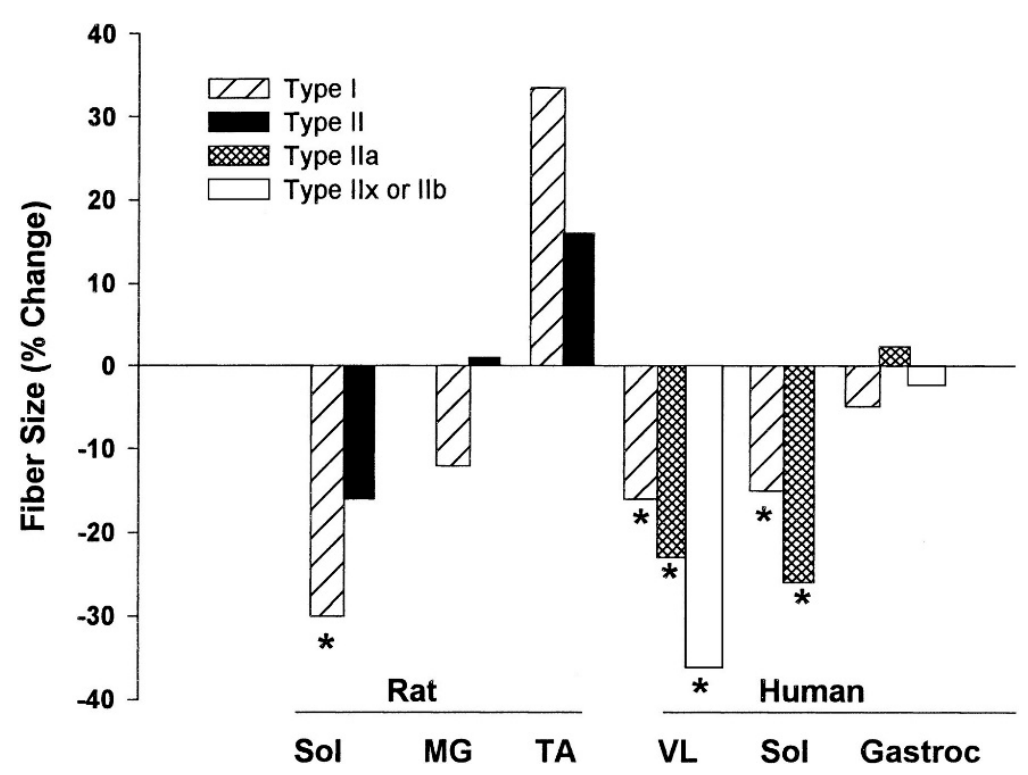

Fig. 1. Percent change in fiber cross-sectional area preflight compared with postflight for slow type I and fast II fibers from the rat soleus (Sol), medial gastrocnemius (MG), and tibialis anterior (TA) and from the human vastus lateralis (VL), Sol, and gastrocnemius (Gastroc). Rat Sol, MG, and TA data are from the 14-day Cosmos 2044 flight (Refs. 59,$\underline{41}$, and $\underline{41}$, respectively). Human VL data are from an 11-day flight (22), whereas the Sol and Gastroc data are from the 17-day STS-78 flight (Ref. $\underline{99}$ and Widrick, Knuth, Norenberg, Romatowski, Bain, Riley, Karhanek, Trappe, Trappe, Costill, and Fitts, unpublished observations, respectively). ${ }^{*} P<0.05$.

Skeletal muscle mass in humans.

Until recently, the only evidence on the extent of human limb muscle atrophy came from gross

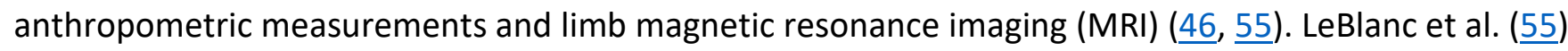
used MRI to assess leg and back muscle volumes before and after an 8-day SF. They observed equal volume losses for the calf and quadriceps (-6.3 and $-6.0 \%)$, whereas the anterior compartment of the lower leg showed less loss (-3.9\%). The hamstrings and intrinsic lumbar muscles underwent the largest declines of -8.0 and $-10.3 \%$, respectively. Studies of Mir cosmonauts after 6 mo of SF showed declines in the calf plantar flexors varying from 6 to $20 \%$ (102). These data and functional results to be described below (see sf effects on skeletal muscle contractile function) illustrate that considerable individual variation exists. Up to now, it has been impossible to determine how much of the variability 
reflects true individual differences as opposed to effects caused by different types and/or degrees of exercise countermeasure or different flight durations. Two recent human studies have evaluated cell size changes in response to 5,11 , and 17 days of $\operatorname{SF}(\underline{22}, \underline{99})$. Consistent with the rat data, fibers within the Sol were more affected than gastrocnemius fibers (Fig. 1). However, unlike in rats exposed to microgravity, type I fibers were not more susceptible to atrophy than type II fibers. Edgerton et al. (22) observed a trend for fiber atrophy after 5 days in space and significant reductions in fiber crosssectional area (CSA) after an 11-day flight with type IIb > Ila >I (Fig. 1). Widrick et al. (99) reported a similar finding for the Sol, for which, after a 17-day SF, type lla fiber CSA declined $26 \%$ compared with a $15 \%$ reduction in the slow type I fiber (Fig. 1). The SF-induced atrophy was particularly evident when myofibrils were analyzed at the electron microscope level $(\underline{76}, 99)$. The postflight Sol type I fiber atrophy is reflected by the $39 \%$ decrease in Z-band length and thinner myofibrils in the post-compared with the preflight longitudinal electron microscope sections (Fig. 2). This study also demonstrated the considerable subject variability that exists in response to SF. The percent decline in fiber diameter in the slow type I fiber of the Sol in four crew members ranged from 2 to $19 \%$ (99).

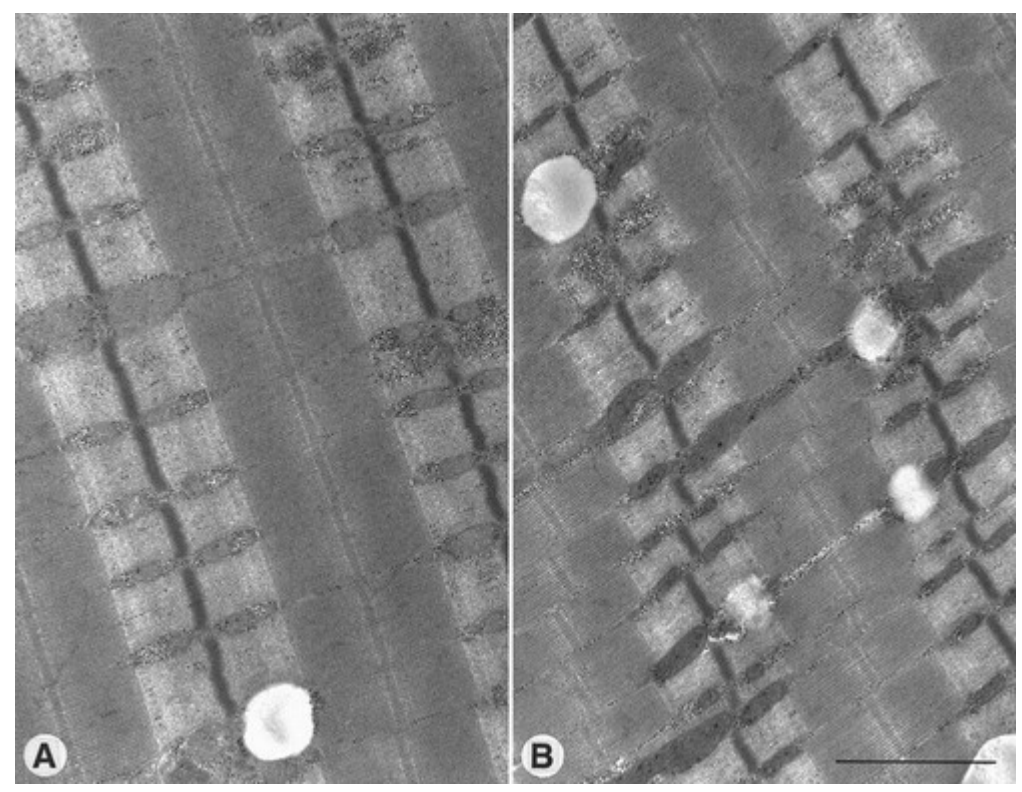

Fig. 2. Electron micrographs of longitudinal sections of slow muscle fibers obtained from soleus muscles of subject $C$ before $(A)$ and after $(B)$ a 17-day spaceflight (99). Preflight control fiber has wide myofibrils, whereas myofibrils postflight are thinner, indicating atrophy. Mitochondria and glycogen-like granules are similar in both fibers, but lipid droplets are more frequent postflight. Bar $=1.5 \mu \mathrm{m}$.

One explanation for the greater microgravity-induced atrophy of the type lla fiber in humans and the type I fiber in rats may relate to the average preflight fiber size. In rats, the type lla fiber type is considerably smaller than the type I fiber, whereas in humans the type lla fiber is slightly larger than the type I fiber (31). In their review paper, Edgerton and Roy (21) commented that one consistent observation was that the greater the preflight fiber size the greater was the degree of atrophy for a given fiber type postflight. This generalization appears to hold within a species and fiber type as well. In the Widrick et al. (99) study, the astronaut with the greatest type I fiber atrophy had the largest average fiber size preflight. 


\section{QUALITATIVE AND QUANTITATIVE ALTERATIONS IN CELL PROTEINS}

\section{Fiber type.}

The fiber-type changes in response to SF in rats has recently been reviewed by Edgerton and Roy (21). The available data indicate that, within the first week of SF, the number of slow fibers in the antigravity muscles of the rat decreases, whereas the number of fibers containing fast-type myosin increases. For example, after a 7-day flight, Martin et al. (ㅌ) observed a 39-50 and a $20-46 \%$ increase in the number of dark ATPase (fast) fibers in the Sol and AL muscles, respectively. In contrast, the 7-day SF had no effect on the percent fiber type distribution in the fast plantaris and superficial region of the MG (extensor muscles) or the fast EDL (a flexor muscle). Longer flights of 12.5 and 14 days showed the same result: an increased number of fast fibers in the slow antigravity muscles with no alterations in the fast-twitch muscles $(\underline{47}, \underline{63}, \underline{68})$. Ohira et al. (요) reported that the percentage of Sol fibers showing a positive reaction with both slow and fast myosin heavy chain (MHC) antibodies after a 14-day SF increased from 0 to $16 \%$, whereas those reacting only to slow MHC declined from 90 to $76 \%$. Thus SF in rats increased the number of hybrid fibers in antigravity slow muscles by inducing fast myosin expression in slow fibers, whereas it had little or no effect on the number of pure fast fibers. The data of Caiozzo et al. (12) suggest that the increased number of hybrid fibers may be caused by type Ilx myosin expression because a 6-day SF increased this isozyme, whereas the type lla isoform significantly decreased. Haddad et al. (39) expressed the MHC type as a percentage of the total myosin pool. After a 9-day SF, they observed a trend for the type I MHC to decrease in both the vastus intermedius (VI) and the deep red region of the vastus lateralis (RVL). However, the only significant change was a decline in $\mathrm{IIa/IIx} \mathrm{MHC} \mathrm{in} \mathrm{the} \mathrm{RVL} \mathrm{from} \mathrm{the} \mathrm{control} \mathrm{value} \mathrm{of} 61.1 \%$ to the postflight value of $50.7 \%$. In this muscle there was a corresponding increase in the IIb MHC from a control value of $29.2 \%$ to the postflight value of $42.9 \%$. The antibody used could not separate the type lla and IIx MHCs, but it seems likely, on the basis of the data of Caiozzo et al. (12), that the decline in the Ila/IIx MHC was caused by a drop in the Ila and not the IIx myosin.

In humans, Edgerton et al. (22) reported an 11-day SF to decrease the slow type I fiber from 48 to $40 \%$, whereas it increased the fast type lla from 32 to $41 \%$ in the vastus lateralis (VL). They observed no change in the percentage of fast type llb fibers (20\% preflight vs. $19 \%$ postflight).

Immunohistochemical and SDS-gel electrophoresis techniques have demonstrated that human muscle contains little or no type Ilb myosin $(\underline{23}, \underline{83})$. Thus the histochemically identified type Ilb fibers in the Edgerton et al. study were likely type Ilx fibers. Recently, Widrick et al. (99) studied the effects of a 17day SF on the properties of single fibers isolated from the Sol. On average, $91 \%$ of the pre- and $79 \%$ of the postflight fibers expressed type I MHC. The results indicated a significant SF-induced decline in the percentage of fibers expressing type I MHC and a corresponding increase in fibers containing type $\mathrm{I} / \mathrm{Ila}$ or Ila MHC. Similar to fiber CSA, considerable subject variability existed for MHC expression. The reduction in the expression of type I MHC could be attributed entirely to the responses of two of the four crew members studied.

\section{Total, myofibrillar, and sarcoplasmic protein content.}

As reviewed above, rats flown in space show a rapid decline in the mass of antigravity muscles such as the Sol, and thus one would expect to see a similar drop in total protein. Consistent with this, Steffen and Musacchia (84) observed a significant decline in both myofibril and sarcoplasmic protein after a 7- 
day SF (Skylab 3) in the Sol and gastrocnemius but not in the EDL. In the Sol, the myofibril protein loss was greater than that observed for the sarcoplasmic proteins. The protein concentration of these muscles was similar to the 1-G controls, which indicates that the protein loss mirrored the decline in muscle mass. Haddad et al. (푸) observed similar results after a 9-day SF in which the VI showed significant atrophy but no change in the total or myofibril protein concentration in milligrams per gram of tissue. The authors used the muscle weight, myofibril yield $(\mathrm{mg} / \mathrm{g})$, percentage of myosin in myofibril pool, and relative percentage of $\mathrm{MHC}$ isoform in the myosin pool to calculate the content of each myosin isozyme. In the control VI, there was on average 1.72 and $1.40 \mathrm{mg} / \mathrm{muscle}$ of type I and IIa/IIx $\mathrm{MHC}$, respectively, whereas in the flight VI there was $1.04,1.13$, and $0.05 \mathrm{mg} / \mathrm{muscle}$ of type I, Ila/IIx, and IIb MHC, respectively. These data suggest that the decline in myofibril protein can be attributed to a loss of the slow type I myosin and, to a lesser extent, of the fast type Ila/Ilx myosin. As reviewed in Fiber typeabove, the latter likely reflects a loss in the lla rather than the Ilx protein.

In contrast to 7 days of SF, by 12.5 days a significant decline in myofibril yields (mg protein/g muscle) was observed in the slow-twitch VI but not the fast-twitch VL (4)). The VI myofibril yield declined from a control value of 108 to a postflight value of $71 \mathrm{mg} / \mathrm{g}$. On the basis of densitometric analysis of gel bands of native myosins separated by pyrophosphate electrophoresis, the authors concluded that the loss of total myosin in the VI muscle of the flight group was attributed to reductions in the slow and intermediate (presumably type Ila) myosin isozymes (4)). Interestingly, even though there was no loss in total myosin content in the VL postflight, this muscle showed a drop in intermediate myosin that was balanced by an increased fast (presumably type IIx and IIb) myosin. Clearly, in rats, muscle wasting is associated with a selective loss of slow-type myosin protein. In humans, there are no published data on protein content. However, the loss of force per CSA in slow type I fibers and the increased number of fast type Ila fibers suggest that slow-type myosin was also selectively lost in humans $(\underline{22}, \underline{99}, \underline{103})$.

\section{Mechanisms responsible for the myofibril protein loss.}

Baldwin et al. (4) suggest that the early decline in myofibril yields expressed on a muscle basis ( $\mathrm{mg} / \mathrm{g} \times$ muscle weight) indicates that myofibril degradation is an early event in the atrophy of rat hindlimb muscles in response to $0 \mathrm{G}$. However, rat $\mathrm{HU}$ studies indicate that the earliest event in Sol muscle atrophy was a decrease in myofibril synthesis (92). Thus, for the first few days of HU, the protein loss was attributed almost entirely to a reduced synthesis. From day 3 on, the synthesis rate remained steady, whereas the degradation rate showed a large transient increase. The large majority of the protein loss after the first few days was attributed to the increased degradation rate (92). Data from both SF and bed rest suggest that the primary and perhaps exclusive mechanism for the loss of muscle proteins in humans is a decline in synthesis. Ferrando et al. (26) studied this question by using the simulated microgravity model of $-6^{\circ}$ head-down bed rest. After 14 days of bed rest, they observed an $\sim 50 \%$ drop in protein synthesis with no change in protein breakdown in the VL muscle. The authors found no change in serum cortisol, testosterone, insulin-like growth factor I (IGF-I), or insulin and concluded that the decrease in protein synthesis could not be explained by hormonal alterations. These data are supported by the observation of LeBlanc et al. (ㅎ6), who found 17 wk of bed rest to have no effect on the level of 3-methylhistidine excretion, an indicator of muscle breakdown.

Additionally, the data of Criswell et al. (16) suggest that muscle atrophy in HU mice was not caused by a decline in IGF-I mRNA expression and that transgenic mice overexpressing human IGF-I were not protected from $\mathrm{HU}$-induced muscle atrophy. 
Recently, prolonged SF of $>3$ mo on Mir was shown to reduce whole body protein synthesis rates by $45 \%$ in astronauts and cosmonauts (6). Interestingly, estimates of whole body protein breakdown demonstrated a reduction in breakdown that paralleled the reduced synthesis (ㅎ6). For the six subjects studied, the decrease in protein synthesis was significantly correlated to a decreased energy intake. Stein et al. (8ㅏ) evaluated whether the decline in muscle protein could be in part explained by an altered hormonal status. Urinary hormones before, during, and after a 9.5-day [Spacelab Life Science (SLS)-1] and 15-day (SLS-2) SF were assessed. With the exception of cortisol, which showed a small increase, there was no effect of flight on the urinary excretion of the hypothalamic-pituitary-adrenal (HPA) axis hormones, including growth hormone (GH). These results led the authors to argue against a primary role for the HPA axis and GH in particular in the regulation of muscle protein content inflight. The increased cortisol agreed with earlier data collected on Skylab and confirms that SF likely does increase serum cortisol. However, the authors argue that the increase does not play a major role in the SF-induced muscle protein loss (8ㅗ). They point out an increased cortisol would have systemic affects, which could not explain the selective atrophy of antigravity muscles. Additionally, cortisol primarily stimulates protein breakdown, and there was no evidence on these flights or the Mir experiments that myofibril protein breakdown increased. Collectively, these data suggest that, at least for humans, the primary mechanism for the decline in myofibril protein content after both SF and bed rest is a decline in protein synthesis. The data of Stein and colleagues from both the Mir mission ( $\underline{86}$ ) and shuttle flight STS-78 (그) suggest that the decline in protein synthesis may be exacerbated by a negative energy balance.

Recently, considerable effort has been extended toward the elucidation of the molecular changes responsible for the reduced protein synthesis and muscle atrophy associated with unloading $(\underline{13}, \underline{17}, \underline{59}, \underline{60}, \underline{91})$. In rats, it is well established that both HU and SF cause a selective loss of contractile protein and slow-to-fast transitions in contractile and regulatory proteins $(\underline{24}, \underline{59})$. The reduced protein synthesis appears to result from changes in transcription and translation ( $\underline{53}, \underline{59}, \underline{60})$. Criswell et al. (17) observed 7 days of HU in mice to significantly decrease Sol troponin I slow (Tnls) mRNA, whereas the fast troponin I (TnIf) mRNA showed a tendency to increase. The authors concluded that the decrease in the ratio of Tnls to TnIf resulted from a large downregulation of the Tnls gene, whereas the TnIf gene expression was maintained at the control level. The latter would seem to be unlikely in humans in whom type lla fiber atrophy with SF was equal to or larger than that observed for the slow type I fiber $(\underline{22}, \underline{99})$. The answer to the question of whether fast and slow isozymes are regulated independently, and the effect that such regulation has on function, will require molecular and physiological analyses of individual single fibers.

Thomason et al. (92) reported that the $\beta-\mathrm{MHC}$ mRNA concentration of the Sol did not decrease with 7 days of HU. From this they concluded that the rapid decrease in myofibril synthesis was unlikely to be transcriptionally driven. More recently, Ku and Thomason ( $\underline{53}$ ) showed that polysomes isolated from Sol muscles of rats suspended for $18 \mathrm{~h}$ were larger (more ribosomes per mRNA) than polysomes from control animals. The authors concluded that the increased polysome size was caused by a slowing of the nascent polypeptide chain elongation. This in turn slowed the translation rate, reducing protein synthesis. The 70-kDa heat shock protein (HSP70) is thought to associate with the nascent polypeptide and facilitate protein translation. Recently, Ku et al. (54) observed a 44 and $28 \%$ decrease in HSP70 proteins associated with polysomes after 12 and $18 \mathrm{~h}$ of $\mathrm{HU}$, respectively. They suggested that a HU- 
induced increase in cellular ATP may have enhanced the dissociation of HSP70 proteins from the polysomes, thus slowing ribosome translocation and elongation rate.

Although Thomason et al. (92) found no decrease in Sol $\beta-M H C$ mRNA with 7 days of HU, McCarthy et al. (9) observed a significant decline in this message by 14 days in the mouse Sol. Their results indicated that a 600-bp region of the promoter sequence was sufficient to direct the decreased transcription of $\beta-\mathrm{MHC}$ transgenes in response to $\mathrm{HU}$. One complication in the interpretation of this result was the finding that $\mathrm{HU}$ coupled with ablation of the synergistic muscles prevented Sol atrophy but not the depressed transgene expression. In a recent paper, McCarthy et al. (60) found that distinct $\beta-\mathrm{MHC}$ promoter sequences mediate $\beta-\mathrm{MHC}$ expression in response to overload and unloading.

Collectively, these data suggest that overload of the Sol during HU was able to prevent muscle atrophy by overriding the unloading-induced inhibition of the promoter. A second important observation from this work was that a negative regulatory element may be involved in the decreased $\beta-\mathrm{MHC}$ expression (60). The increased numbers of hybrid and fast fiber types after $\mathrm{HU}$ in rats and mice and SF in rats and humans suggest not only a downregulation of the $\beta-\mathrm{MHC}$ gene but also an upregulation of fast-type myosin isoforms. This hypothesis was supported by the data of Swoap (91), who reported no detectable IIb myosin mRNA in control Sol but a level for this message of $\sim 10 \%$ of that of the TA in the Sol after $3 \mathrm{wk}$ of $\mathrm{HU}$. The data also showed that a 295-bp proximal upstream regulatory region of the $\mathrm{MHC} \mathrm{Ilb}$ gene contained at least one element that was responsive to $\mathrm{HU}$ in the Sol.

Although 7 days of $\mathrm{HU}$ did not alter the $\beta$-myosin MHC mRNA, this model as well as SF have been shown to decrease $\alpha$-actin mRNA $(\underline{92}, \underline{93})$. By 7 days of HU, Babij and Booth (ㅇ) reported a 60 and $29 \%$ decline in Sol $\alpha$-actin mRNA expressed per muscle and per microgram RNA, respectively. After a 14-day SF, rat skeletal muscle $\alpha$-actin mRNA per unit of extractable RNA decreased 25 and $36 \%$ in the $\mathrm{VI}$ and lateral gastrocnemius muscles, respectively. This suggests that myosin and actin content are regulated independently and that their synthesis rate during SF and models of weightlessness are controlled by different mechanisms. Consistent with this hypothesis was the recent finding that a 17-day SF (STS-78) induced a disproportionate loss of actin filaments in human Sol muscle. Thick-filament density and spacing were unchanged, whereas thin-filament density decreased significantly in the overlap A-band region (99). The decline was the result of a $17 \%$ filament loss and a $9 \%$ increase in short filaments that failed to penetrate the A band (Fig. 3 ). The thin filament loss was calculated to increase the thick-tothin filament spacing in vivo from 17 to $23 \mathrm{~nm}(\underline{76})$. The authors hypothesized that this structural change was responsible for the $\sim 30 \%$ postflight increase in the maximal unloaded shortening velocity $\left(V_{0}\right)$ and for a reduced peak stiffness in the slow type I fiber ( $\left.\underline{99}\right)$. 
Normal

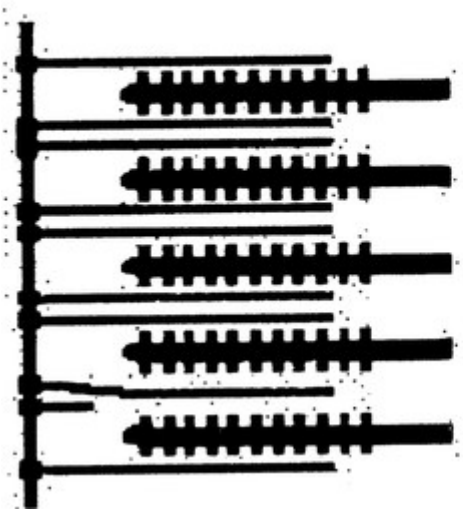

\section{Atophic}

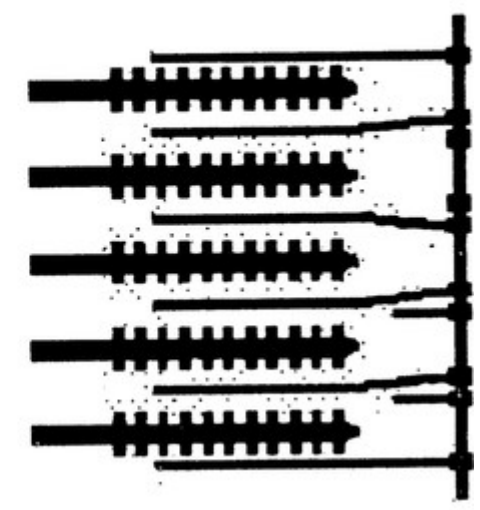

Fig. 3. Schematic representation of in vivo status of thin-filament packing density and spacing in one-half of a sarcomere of a normal preflight muscle and in one-half of a sarcomere from an atrophic muscle after a 17-day spaceflight in humans. For the preflight controls, $13 \%$ of thin filaments were not long enough $(<0.5 \mu \mathrm{m})$ to penetrate the $\mathrm{A}$ band in a $2.4-\mu \mathrm{m}$ sarcomere. Subsequent to atrophy after spaceflight, short thin filaments increased by $9 \%$, and $17 \%$ of the thin filaments were lost. These changes summated to produce a $26 \%$ decrease in thin filament density in overlap A-band region. Reduced thin filament density was calculated to increase the thick-to-thin filament spacing by $35 \%$, which is postulated to cause an earlier detachment, less drag, and faster cycling of cross-bridges, leading to an increased maximal shortening velocity in the postflight fibers $(\underline{62}, \underline{76}, \underline{99})$. [Redrawn from Riley et al. (흐).]

\section{SF EFFECTS ON SKELETAL MUSCLE CONTRACTILE FUNCTION}

\section{Muscle force.}

Since the Skylab missions in the early 1970s, it has been known that SF reduces the peak force of limb skeletal muscles $(\underline{15}, \underline{35})$. Skylab 2 (28-day mission) showed a greater drop in thigh vs. arm and extensor vs. flexor torque, with the peak extensor torque of the thigh declining by $20 \%$ compared with a $10 \%$ loss in thigh flexor and arm extensor groups. Skylab 3 (59-day mission) showed an even greater difference between the SF-induced loss in thigh (mean 20\% drop) and arm torque (mean $2 \%$ drop) than Skylab 2. The differences were ameliorated on Skylab 4 (84-day mission), in which the leg exercise countermeasures were credited with reducing the mean loss of peak thigh torque to $6 \%(\underline{35})$. In longer Mir flights of 6-mo duration, three cosmonauts showed a $20-48 \%$ decline in the maximal voluntary contraction (MVC) of the calf plantar flexion (102). The reduced MVC exceeded the percent loss of calf muscle volume determined by MRI, and a correlation between the decline in muscle volume and MVC was not found. Goubel ( $\underline{33}$ ) also observed the MVC of the calf during plantar flexion to decrease after 1,3 , and 6 mo aboard the Mir, with a range of change from 0.1 to $37.6 \%$. However, the data showed no clear relationship between flight duration and the fall in MVC.

Greenleaf et al. (35) reviewed the effects of bed rest and SF on the percent change of TA (ankle dorsiflexion) and triceps surae (ankle plantar flexion) strength measured isokinetically at four velocities $\left(0,60,120\right.$, and $\left.180^{\circ} / \mathrm{s}\right)$. A SF duration of 7 days had no significant effect on either muscle group at any of the four velocities tested. In contrast, after 237 days in space, both ankle dorsiflexion and plantar flexion strength were significantly reduced at all velocities ( $\underline{35})$. Figure $\underline{3}$ of the Greenleaf et al. $(\underline{35})$ review, which was redrawn from the data of Grigor'yeva and Kozlovskaya ( $\underline{37})$, is reproduced here (Fig. 4). The figure demonstrates two important observations: 1) 110 days of microgravity reduced 
ankle plantar flexion strength as much as 237 days, and 2) prolonged SF ( $>110$ days) reduced the peak torque of the dorsiflexor and plantar flexor muscles to approximately the same amount. The former observation suggests that by 110 days in space a new microgravity steady state has been obtained, such that flights beyond this duration would not be expected to elicit additional declines in peak limb muscle torque. This conclusion is supported by the recently published data of Antonutto et al. (2) that demonstrated peak force during two-legged extension to decline by 11,26 , and $29 \%$ after 31,169 , and 180 days in microgravity, respectively. The second observation is consistent with leg volume measurements that demonstrated a similar degree of muscle wasting in the dorsiflexor and plantar flexor groups of Mir cosmonauts after 4-6 mo in space (A. D. LeBlanc, personnel communication). Collectively, the limb muscle torque data from Skylab 2, Skylab 3, and Mir indicate that the leg extensors lose torque more rapidly than flexors, but by 4 mo little difference exists between these groups. The data of Antonutto et al. indicate that the rate of force recovery is related to the time in space. After a 31-day SF, two-legged peak force was recovered by 6 days postflight, whereas, after 169-180 days in space, peak force was still depressed by $12-22 \%$ after 26 days of recovery.

A

B

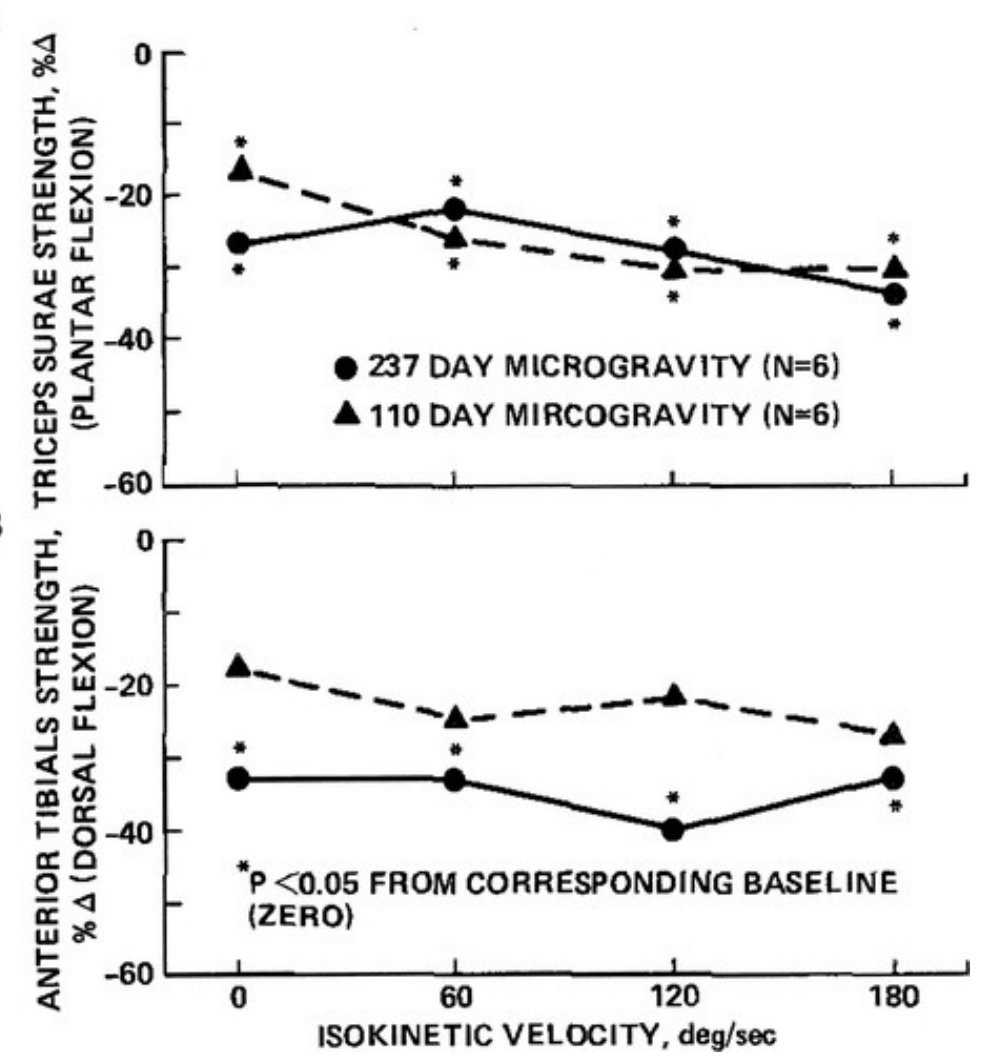

Fig. 4. Comparison of mean percent changes $(\% \Delta)$ in plantar flexion and dorsiflexion isokinetic strengths $\left(0-180^{\circ} / \mathrm{s}\right)$ between 110 and 237 days of exposure to microgravity. $N$, no. of subjects. [Reprinted from Greenleaf et al. ( $\underline{35})$, which had originally been redrawn from Grigor'yeva and Kozlovskaya (37).]

Recently, Widrick et al. (⒐9) determined the peak force of individual slow type I and fast type lla fibers of the Sol in four participating crew members obtained from biopsies taken before and immediately after a 17-day shuttle space flight (STS-78). The average peak force dropped by $21 \%$ in the type I fiber (from $0.99 \pm 0.03$ to $0.78 \pm 0.02 \mathrm{mN}$ ) and by $25 \%$ in the type lla fibers (from $1.41 \pm 0.14$ to $1.06 \pm 0.03$ $\mathrm{mN}$ ). The single fibers from the gastrocnemius (both type I and Ila) were less affected than the Sol 
fibers (J. J. Widrick, S. T. Knuth, K. M. Norenberg, J. G. Romatowski, J. L. W. Bain, D. A. Riley, M. Karhanek, S. W. Trappe, T. A. Trappe, D. L. Costill, and R. H. Fitts, unpublished observations). When the Sol type I fiber peak force was analyzed for individual crew members, considerable differences were apparent in the susceptibility to SF. For example, the decline in peak force ranged from a low of $12 \%$ to a high of $40 \%$. For the type I fiber, peak force declined out of proportion to the loss in fiber CSA, resulting in an average $4 \%$ drop in the peak force per CSA. SF had no effect on the type lla fiber force per CSA. The most likely explanation for the decline in peak force per CSA was a loss in myofibril content. Although myofibril content was not measured, the postflight Sol type I fibers had significantly less peak stiffness, which is consistent with a decline in the number of strongly bound cross bridges per unit fiber area.

Narici et al. (65) studied in vivo muscle function on the same flight (STS-78). Although the MVC of the plantar flexors was not significantly altered during SF, the peak tetanic torque elicited by $50-\mathrm{Hz}$ direct electrical stimulation showed a progressive decline during flight ( $10 \%$ loss by flight day 16) that continued during the first 8 days of recovery. The authors hypothesized that the force drop during recovery was the result of fiber damage after reloading. The single-fiber data of Widrick et al. (99) and the in vivo direct electrical stimulation data of Narici et al. (ㅌ5) are in good agreement and collectively suggest that significant plantar flexor force is lost by 17 days in space, and that the Sol contributes more to the decline than the gastrocnemius.

An interesting question is why SF induces greater atrophy and force decline in the Sol compared with the gastrocnemius. Recent experiments evaluating the effect of a 14-day SF in rhesus monkeys may shed light on this question. Recktenwald et al. (4) demonstrated that two flight monkeys (357 and 484) showed a decreased cycle period and electromyogram (EMG) burst duration of the primary extensors (Sol and MG) during locomotion postflight, whereas the burst amplitude of the TA (flexor muscle) increased. Importantly, probability density distributions of EMG amplitude of the Sol and MG showed a shift toward a higher activation of the MG relative to the Sol post- compared with preflight (Fig. $\underline{5})$. Recktenwald et al. (4) also reported that the MG tendon forces were considerable greater post-compared with preflight during locomotion. We studied the same two monkeys and found greater fiber atrophy and force loss in the Sol type I fiber in monkey 357compared with monkey 484 (29). Of particular importance was the observation that, inflight, monkey 357 showed a progressive change from predominately Sol activity to mainly MG activity over the duration of the flight. In contrast, the Sol/MG recruitment ratio for monkey 484 was unaltered by SF (J. A. Hodgson personal communication). Clearly the Sol type I fiber atrophy and reduced functional capacity in monkey 357 was associated with reduced activation of this muscle. Recktenwald et al. (74) concluded that microgravity induced a reorganization of motor recruitment to favor fast vs. slow motor units and flexors vs. extensor muscles. An unanswered question is whether the altered neuronal recruitment pattern was a direct effect of the microgravity environment (perhaps resulting from a reduced muscle afferent input to the central nervous system) or occurred in response to the Sol muscle atrophy. The observation that the EMG shift occurred inflight, in which atrophy and reduced force production of the Sol would not likely compromise movement or require additional gastrocnemius activation supports the former (74). In either case, the greater microgravity-induced atrophy of the Sol compared with the gastrocnemius has been observed in humans, nonhuman primates, and rodents and can be attributed both to the removal of the antigravity load-bearing role and to the reduced activation of the Sol. 


\section{Pre-flight}

$\# 357$

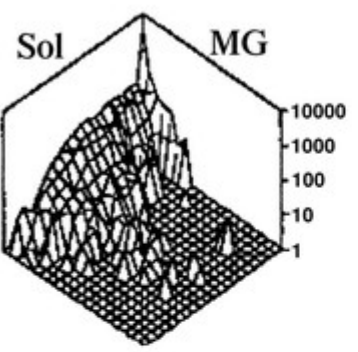

\section{Post-flight}

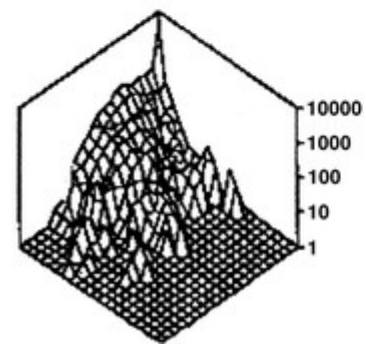

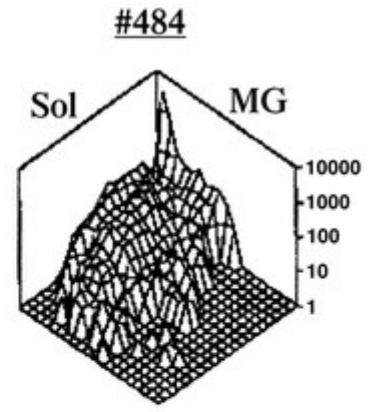

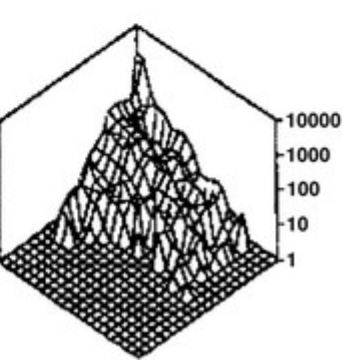

Fig. 5. Probability density distributions of the EMG amplitudes in the Sol and MG muscles pre- and postflight (1 day of recovery) for 2 flight monkeys flown on the 14-day Bion 11 mission. Data were recorded during a sequence of steps at 0.45 $\mathrm{m} / \mathrm{s}$. Horizontal axes show EMG amplitudes with the origin at the back of the plot, obscured by data. Vertical axes are a logarithmic scale of probability [log (1+ probability)]. [From Recktenwald et al. (74).]

The hypothesis that microgravity causes a fundamental alteration in motor control has also been suggested by Antonutto et al. (1). They observed two-legged muscle power to decline considerably more than could be explained by the loss in muscle mass. Additionally, the loss of explosive leg power was associated with a substantial reduction in the EMG activity of the rectus femoris, VL, and vastus medialis muscles. These authors concluded that microgravity induced a basic change in motor control and coordination such that motor activation of extensor muscles was reduced. An increased bias toward flexor activation would help explain why anterior compartment muscles such as the TA generally show little or no atrophy in rats (Fig. 1) and a slower time course for atrophy in humans (ㅁ5).

Compared with humans, rats appear to show a faster decline in limb muscle force in response to SF. Caiozzo et al. $(\underline{11}, \underline{12})$ studied the Sol in situ and observed a 28 and $36 \%$ decline in peak tetanic tension after 6 and 14 days of SF, respectively. These data confirmed earlier observations by Oganov and colleagues $(\underline{66}, \underline{67})$, who found the in situ tetanic force of the Sol to drop 22 and $~ 30 \%$ after 20.5 days (Cosmos 690 biosatellite) and 22 days (Cosmos 605 biosatellite) of SF, respectively. In contrast, the peak force of the EDL muscle showed no significant change after either flight.

The greater susceptibility of rat muscle to SF compared with human muscle was substantiated by single fiber studies carried out by Mounier and colleagues (43, 90). In a 7-day SF (Cosmos 1667 biosatellite), Holy and Mounier ( $\underline{43}$ ) found the peak force of Sol single fibers to decrease by $28 \%$, whereas fibers from the fast gastrocnemius were not significantly altered. However, in a 5-day SF (Cosmos 1514 biosatellite), the gastrocnemius fibers did undergo a significant $22 \%$ drop in peak force. In both the 5 and 7-day flights, the reduced force could be explained by fiber atrophy because the force per CSA was 
unaltered by SF. Fibers from the plantaris muscle showed no significant change after either 5 or 7 days of SF (43). After a 14-day SF (Cosmos 2044 biosatellite), single fibers from the rat Sol and gastrocnemius showed even greater atrophy and loss of force (미). In Sol fibers typed as slow twitch, peak force was reduced to $25 \%$ of the preflight value. The decline was for the most part caused by fiber atrophy because the force per CSA was not significantly altered by flight. As with the shorter flights, the gastrocnemius fibers were less affected than the Sol fibers, and, consistent with the whole muscle experiments, single EDL fibers were not altered by SF (이). In two 18.5-day flights (Cosmos 936 and 1129 biosatellites), Oganov et al. ( $\underline{67}$ ) and Rapcsak et al. (구) found the peak tension of Sol fibers to decline by up to $45 \%$ of the synchronous control group, whereas EDL fibers were unaltered. These authors also found a significant drop in the peak force of fibers isolated from the triceps and brachialis muscles of the forelimb.

An additional factor affecting fiber force is the $\mathrm{Ca}^{2+}$ sensitivity of the fiber. Holy and Mounier ( $\left.\underline{43}\right)$ observed that individual rat Sol fibers postflight (7-day SF) required slightly higher $\mathrm{Ca}^{2+}$ concentrations for activation threshold [- $\log \mathrm{Ca}^{2+}$ concentration (pCa) 6.40 postflight compared with pCa 6.60 preflight], and the $\mathrm{Ca}^{2+}$ concentration required for one-half peak force ( $\mathrm{pCa}_{50}$ ) was also slightly increased. However, the data are inconclusive because no statistical analyses were performed and the fibers were not typed as slow or fast twitch. Thus the slight right shift in the force-pCa relationship may have resulted from an increase in the number of fast fibers postflight. In a subsequent study by the same group in which the fibers were typed, the authors found a 14-day SF to have no significant effect on the slow Sol fiber activation threshold or $\mathrm{pCa}_{50}(\underline{90)}$. We recently studied the effect of a 17-day SF on the force-pCa relationship of single Sol type I fibers isolated from four astronauts (99). The flight had no significant effect on fiber activation threshold or the slope of the relationship either below or above $\mathrm{pCa}_{50}$. However, in the astronaut with the greatest fiber atrophy, the slow Sol fibers required $29 \%$ more free $\mathrm{Ca}^{2+}$ to obtain half-maximal activation postflight. Thus SF does, in some individuals, shift the force-pCa relationship of the type I fiber to the right, but the fibers are still considerably more sensitive to $\mathrm{Ca}^{2+}$ than fast-twitch fibers. The observation that the slope of the force-pCa relationship was unaltered indicates that SF does not alter the cooperativity in the $\mathrm{Ca}^{2+}$ activation of force.

Isometric twitch duration.

The isometric twitch duration is a reflection of the intracellular $\mathrm{Ca}^{2+}$ transient and, as such, depends on the rate and amount of $\mathrm{Ca}^{2+}$ release from the sarcoplasmic reticulum (SR), the intracellular $\mathrm{Ca}^{2+}$ buffers, and the rate of $\mathrm{Ca}^{2+}$ reuptake by the SR. The functional components of the twitch duration are the time to peak tension (TPT) and the relaxation time. Because of the difficulty in accurately measuring the point at which force returns to the pretwitch baseline, researchers generally measure one-half relaxation time $\left(\mathrm{RT}_{1 / 2}\right)$. To our knowledge, there are no published data on the effects of SF on TPT or RT $1 / 2$ in human muscle. However, Caiozzo et al. (11) studied the rat Sol in situ after a 6-day SF and reported TPT to significant decrease by $8.6 \%$. The $\mathrm{RT}_{1 / 2}$ was reduced but not significantly. After a 14-day SF, the same investigative team found a significant decrease in both the Sol TPT (19\%) and $\mathrm{RT}_{1 / 2}(30 \%)$ (12). The most likely explanation for these data is that SF stimulates SR Ca ${ }^{2+}$ release and reuptake, thus decreasing the duration of the $\mathrm{Ca}^{2+}$ transient and the TPT and $\mathrm{RT}_{1 / 2}$. It is apparent from the data of Caiozzo et al. (11) that the effect on TPT occurs first within 1 wk of SF. 
Although no cellular and molecular studies on SR function have been conducted on flight tissue, the rat $\mathrm{HU}$ model has provided important results on this system. Fitts et al. (으) demonstrated a significant 22 and 39\% decline in the Sol TPT after 7 and 14 days of HU, respectively, with no change in $\mathrm{RT}_{1 / 2}$ at either time point. The 14-day results were confirmed by Schulte et al. (2), who, in addition, reported that both TPT and $\mathrm{RT}_{1 / 2}$ were significantly reduced by 28 days of HU. A Western blot analysis at this time point indicated a $300 \%$ increase in fast $\mathrm{SR} \mathrm{Ca}^{2+}$ pump protein, and an enzymatic analysis revealed a $170 \%$ increase in the $\mathrm{Ca}^{2+}$-dependent SR ATPase activity (82). The increased fast $\mathrm{Ca}^{2+}$ pump mRNA and protein occurred rapidly and was 250 and $110 \%$ higher than control by 4 days of HU. Recently, Peters et al. (69) demonstrated that the HU-induced increase in fast $\mathrm{Ca}^{2+}$ pump mRNA in the Sol was caused by an elevated transcription, which occurred by 2 days of HU. By 10 days of HU, the Sol fast SR pump mRNA was $\sim 75 \%$ of that expressed in the control fast-twitch TA muscle. Despite this, the Sol RT $1 / 2$ was not significantly altered until 28 days of $\mathrm{HU}(\underline{82})$. This suggests that additional factors (perhaps an increased expression of TnIf) were required to elicit the $\mathrm{HU}$-induced decline in twitch duration $(\underline{32}, \underline{95})$.

Kandarian et al. ( $\underline{50})$ found 28 days of $\mathrm{HU}$ to significantly stimulate the T-tubular dihydropyridine (DHP)-receptor mRNA in the Sol to levels indistinguishable from those expressed in the control fasttwitch EDL. Because the DHP receptor is an important protein in the excitation-contraction coupling pathway linking the T-tubular action potential to SR $\mathrm{Ca}^{2+}$ release, an increased receptor density might lead to a faster rate of $\mathrm{Ca}^{2+}$ release. Stevens and Mounier (이) utilized single skinned fibers from the Sol to demonstrate that 15 days of $\mathrm{HU}$ increased the sensitivity of $\mathrm{SR} \mathrm{Ca}{ }^{2+}$ release to caffeine and the rate of SR Ca ${ }^{2+}$ reuptake. The former observation suggests that HU directly affected the SR $\mathrm{Ca}^{2+}$ release channel.

Consistent with the shortened twitch duration, Caiozzo et al. $(\underline{11}, \underline{12})$ found both 6 and 14 days of SF to shift the force-frequency curve of the in situ Sol to the right. After 6 days, significant differences were observed at 5-, 15-, 20-, and 30-Hz stimulation (11). Although a statistical analysis was not carried out, it appeared that the right shift in the force-frequency curve was greater with a 14-day SF than with the 6-day SF (12). Schulte et al. (2) observed a similar response in HU rats: the right shift in the curve was more evident after 28 vs. 14 days of $\mathrm{HU}$.

Fiber maximal shortening velocity and peak power.

Relatively little information exists regarding the effects of SF on the maximal shortening velocity $\left(V_{\max }\right)$ or peak power. However, recently both have been examined in rats and humans $(\underline{11}, \underline{99})$. Caiozzo et al. $(\underline{11}, 12)$ used loaded contractions to calculate $V_{\max }$ of the in situ rat Sol and found 6 and 14 days of SF to increase $V_{\max }$ by 14 and $20 \%$, respectively. The peak power of the Sol calculated from the force-velocity data was significantly reduced after both flights. The decline in power indicates that the postflight increase in muscle velocity was unable to fully compensate for the reduced force-generating capacity of the muscle. The authors suggested that the elevation in muscle velocity might have resulted from the increased percentage of fast myosin [both MHC and myosin light chain (MLC) isozymes] in the slow Sol muscle (12).

Goubel ( $\underline{33})$ studied two astronauts and five cosmonauts who lived on the Mir from 3 to 6 mo. When plantar flexion shortening velocity was measured during a contraction at $15 \%$ of peak power, all but one of the subjects showed an increased velocity postflight. Recently, Widrick et al. (99) evaluated the effects of a 17-day SF on the $V_{\mathrm{o}}$ and $V_{\max }$ of individual slow type I and fast type Ila fibers isolated from 
the Sol and gastrocnemius of four astronauts. In the Sol, the type I fiber $V_{\circ}$ and $V_{\text {max }}$ increased in all four subjects, and the postflight increases in both measurements of shortening velocity were qualitatively similar (30 and 44\%, respectively). The $V_{o}$ and $V_{\max }$ of the Sol type lla fiber also increased postflight, with the largest change observed in $V_{0}$, which increased from $2.90 \pm 0.40$ to $4.49 \pm 0.44$ fiber lengths $/ \mathrm{s}$ or $55 \%$. For the gastrocnemius muscle, the type I fibers showed a $22 \%$ increase in $V_{o}$ after SF. This increase was significant but was less than that observed for the Sol type I fibers. In contrast, the $V_{o}$ of the fast type Ila gastrocnemius fibers was not significantly altered by SF (Widrick, Knuth, Norenberg, Romatowski, Bain, Riley, Karhanek, Trappe, Trappe, Costill, and Fitts, unpublished observations).

The cause of the SF-induced increase in $V_{o}$ and $V_{\max }$ is unknown. The increase could not be attributed to the expression of fast MHCs or MLCs in the slow type I fibers. Although Sol type I fibers from two of the astronauts had an increased $\mathrm{MLC}_{3}$ content, the level of this MLC isoform was not correlated with $V_{0}$ for any of the subjects (⒐9). The authors hypothesized that the increased $V_{\mathrm{o}}$ and $V_{\max }$ might have occurred due to the selective loss of thin filaments (Fig. $\underline{3}$ ). This structural change would be expected to increase the distance between the thin and thick filaments. As a result, the cycling cross bridges would be expected to detach sooner, which in turn would reduce the internal drag that develops during the final portion of the cross-bridge stroke (드).

Mean power-force curves for the Sol type I and Ila fibers are shown in Fig. $\underline{6}$. The plots clearly show the SF-induced drop in peak isometric force (point where the curves cross the $x$-axis) for both the slow and fast fiber types. During contractions eliciting peak power, both fiber types generated significantly less force but shortened at higher velocities such that the peak power obtained was only slightly less than the preflight value (Fig. 6 ) However, in the two astronauts with the largest drop in fiber force, significant declines in peak power of $\sim 20 \%$ were observed in the type I fibers of the Sol (99). The reduced power was entirely explained by the fiber atrophy because the peak power per CSA was unaltered. In contrast to the Sol, SF had no effect on the peak power of either the slow or fast fiber types of the gastrocnemius (Widrick, Knuth, Norenberg, Romatowski, Bain, Riley, Karhanek, Trappe, Trappe, Costill, and Fitts, unpublished observations).

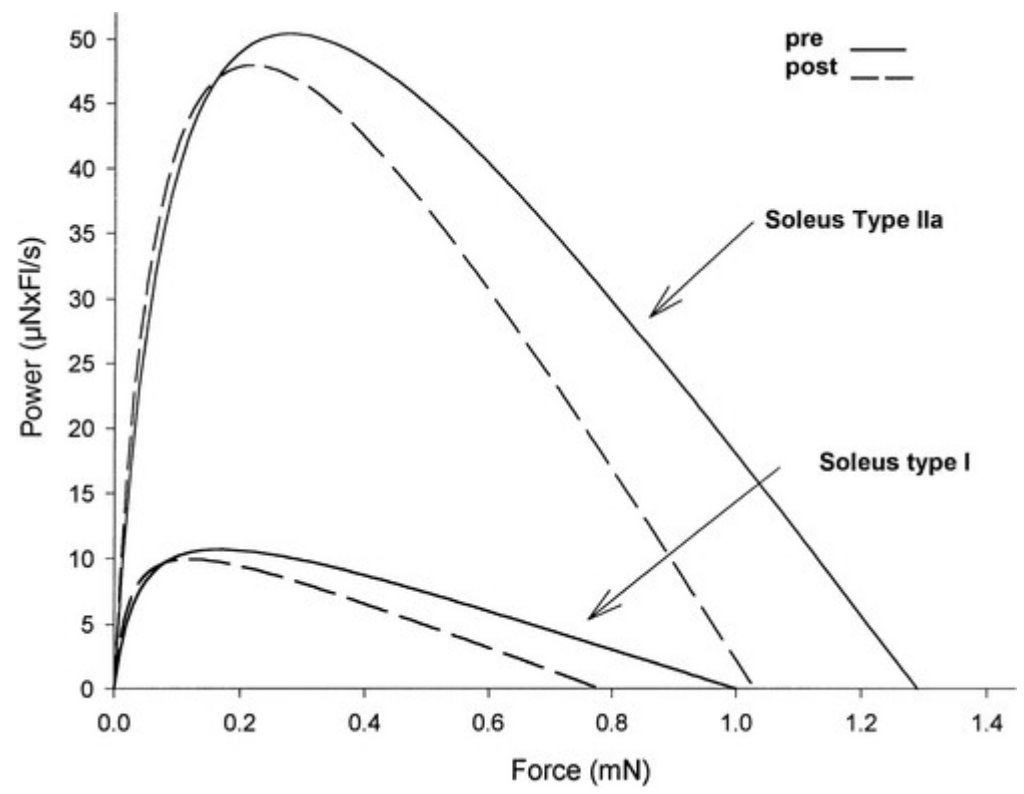


Fig. 6. Mean force-power relationships for preflight (pre) and postflight (post) type I and type lla fibers isolated from the soleus. Plots intercept the $x$-axis at peak isometric force. Plots demonstrate the significantly greater peak power in type Ila vs. type I fibers and the postflight decline in both type I and type lla peak isometric force. Average peak power (mean value for all 4 astronauts) for a given fiber type was unaffected by spaceflight (pre- vs. postflight). Despite this, 2 of the 4

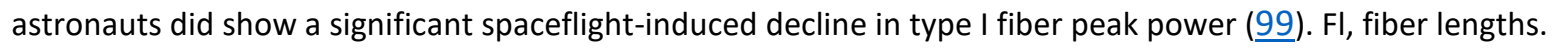

Recently, Antonutto et al. $(\underline{1}, \underline{2})$ examined the effects of SF of from 21 to 180 days on the maximal power of the lower limbs. In the one astronaut studied after 21 days, peak leg power was reduced by $54 \%$. This was considerably greater than the loss in single-fiber power after the 17-day SF and suggests that factors other than fiber atrophy contributed to the change. One possibility suggested by Antonutto et al. (1) and reviewed above was that microgravity altered the motor unit recruitment pattern. In a separate study, Antonutto et al. (2) observed two-legged peak power to drop by $32 \%$ after 31 days and by 50\% after 169-180 days in space. In one astronaut who remained in space for 438 days, the authors calculated that leg power showed a similar decline to that observed at 180 days. Thus the time course for the decline in peak power to a new microgravity steady state was $\sim 6$ mo, a value similar to that observed for muscle mass and force (35).

\section{SUBSTRATE AND METABOLITE CHANGES WITH SF: IMPACT ON FATIGUE}

\section{Muscle substrate alterations with SF.}

$\mathrm{HU}$ in rats and bed rest in humans have been shown to increase Sol muscle glycogen, and Cosmos and Spacelab flights have documented that rat skeletal muscle glycogen increased with SF $(\underline{36}, \underline{64}, \underline{78})$. We know of no published reports on the effects of microgravity on human muscle glycogen. Stein et al. (87) recently published a paper in which they compared the energy expenditure during a 17-day SF and 17-day bed rest. Energy balance was unchanged with bed rest, whereas the four astronauts were found to have an average negative energy balance of 1,355 kcal/day. We studied the same subjects and found that the post-bed-rest Sol muscle fibers showed significant increases in glycogen in both the slow type I and fast type Ila fibers (unpublished data). In contrast, after SF, no significant difference was observed in the Sol type I fiber glycogen (pre- vs. postflight), whereas the Sol type lla fiber glycogen was significantly depressed postflight (unpublished data). The failure to observe an increased Sol muscle glycogen postflight was likely the result of the extremely low inflight dietary intake of $24.6 \pm$ $3.3 \mathrm{kcal} \cdot \mathrm{kg}^{-1} \cdot \mathrm{day}^{-1}(\underline{87})$. As a result of the low caloric input, the astronauts lost on average $2.6 \mathrm{~kg}$ of body weight; however, despite this condition, electron micrographs of the Sol muscle demonstrated a higher content of lipid droplets in the postflight compared with the preflight muscles (99). This observation is consistent with the finding of Musacchia et al. (64), who found an increased triglyceride storage in the rat vastus medialis after a 14-day SF.

\section{Muscle enzymatic alterations with SF.}

Considerable data exist regarding the effects of short-term (4- to 22-day) SF on the oxidative and glycolytic enzymes of rat skeletal muscle (21). The general finding is that the loss of mitochondrial protein with SF is less than that observed for the contractile proteins and for the degree of cell atrophy. As a consequence, the usual observation is for oxidative enzymes of limb skeletal muscle to either remain unaltered or slightly elevated when activity is expressed per gram of dry weight $(\underline{21}, \underline{57})$. This is true for both the slow antigravity muscles such as the Sol and AL and for fast muscles like the TA 
and EDL $(\underline{47}$, 58). For example, no change in succinate dehydrogenase (SDH) activity (per cell volume or gram tissue) of the Sol was observed after 4, 7, 12.5, or 14 days of SF $(\underline{48}, \underline{58}, \underline{63}, \underline{68})$; however, both Miu et al. (즈) and Manchester et al. (그) calculated that the total amount of oxidative enzymes in the Sol was less postflight. Riley and co-workers $(\underline{78}, \underline{80})$ and Bell et al. $(\underline{10})$ showed that mitochondria were selectively lost from the subsarcolemmal regions of the fiber.

Two single-fiber studies evaluating the effect of SF on rat Sol have shown glycolytic enzymes to increase after microgravity. Manchester et al. (ㄱ) found an average increase of $28 \%$ in selected glycolytic enzymes after a 12.5-day SF, and Chi et al. (14) reported pyruvate kinase and glycerol-3phosphate dehydrogenase (GPDH) to increase by 41 and 56\%, respectively, after a 2-wk flight. In contrast to the slow Sol, no significant changes in glycolytic enzymes were observed for fibers isolated from the fast TA. In whole muscle analyses, SF has been reported to have no effect or to increase marker enzymes of glycolysis. For example, after a 7-day SF, Desplanches et al. (20) found no change in Sol lactate dehydrogenase (LDH) activity, whereas Martin et al. (ㅎ8) observed an $\sim 50 \%$ increase in GPDH in the Sol and AL. After 14 days in microgravity, the LDH activity of the rat vastus medialis was increased 52\%, and after 21.5 days the increased LDH activity in the Sol was associated with an increase in the $\mathrm{M}$ form of the enzyme $(\underline{64}, \underline{72})$.

The first enzyme in the processing of glucose after its uptake into skeletal muscle is hexokinase (HK), which converts glucose to glucose 6-phosphate. When single fibers were isolated from the rat Sol and TA muscles after a 12.5-day SF, they showed a $>100 \%$ increase in HK activity (57). When expressed in absolute amounts of enzyme, the HK activity was still increased postflight by 50 and 25\%, respectively, in the Sol and TA. In a second study by the same group, Sol HK activity was found to increase by only 47\% after a 14-day SF, and the TA HK activity was unaltered (14).

Considerable less information is available concerning the effects of SF on enzyme activities in human limb skeletal muscle. Edgerton et al. (22) found no significant affects of an 11-day SF on the SDH or GPDH activities of VL fibers. Although the change was not significant, the slow type I fibers did show an $80 \%$ increase in GPDH activity, and, as a result, GPDH/SDH was significantly higher postflight. Recently, we evaluated the enzyme profile of single fibers isolated from the Sol and gastrocnemius muscles of astronauts before and immediately after a 17-day SF (STS-78). Consistent with the rat data, microgravity-induced a significant increase in the oxidative enzymes of the slow type I fibers of the Sol (unpublished observations). However, surprisingly the Sol type I fiber was unaltered in regard to HK activity or the glycolytic enzymes glycogen phosphorylase, glycogen synthase (GS), phosphofructokinase, or LDH (unpublished observations). The gastrocnemius type I and Ila fibers contained elevated oxidative enzyme activities postflight, and the latter also showed an increased HK, GS, and LDH activity (unpublished observations).

Recently, Kell et al. (1ㅗ) studied the molecular regulation of the muscle-specific form of the glycolytic enzyme phosphoglycerate mutase (PGAM-M) in response to $4 \mathrm{wk}$ of $\mathrm{HU}$ in the rat. PGAM-M enzymatic activity and mRNA in the Sol increased 2.5- and 3.5-fold, respectively, compared with control muscles. The proximal 400-bp region of the PGAM-M promoter was increased two to threefold in the unweighted Sol. These data suggest that transcriptional activation was important in the upregulation of PGAM-M with unloading. 
Substrate utilization and muscle fatigue.

The microgravity environment appears to increase muscle fatigue during work in space and on return to $1 \mathrm{G}(\underline{46})$. Caiozzo et al. (11) stimulated the rat soleus in situ and observed significantly greater fatigue in the flight compared with the control group (Fig. ㄱ). The cause of the increased fatigue is unknown but may relate to an accelerated glycogen utilization and a reduced capacity to oxidize fats. Support for this hypothesis comes from the work of Baldwin et al. (므). After a 9-day SF, they observed a $37 \%$ decline in the ability of both the high-and low-oxidative regions of the rat vastus muscle to oxidize long-chain fatty acids. They found no change in the muscle's ability to oxidize pyruvate or in key marker enzymes of the Krebs cycle or $\beta$-oxidative pathway. The authors hypothesized that the inhibition of long-chain fatty acid oxidation postflight might result from a reduced ability to activate or translocate fats from the cytosol to the mitochondria. The latter process is particularly intriguing as it is catalyzed by carnitine palmitoyltransferase (CPT I), which is thought to be the rate-limiting step in fatty acid oxidation.

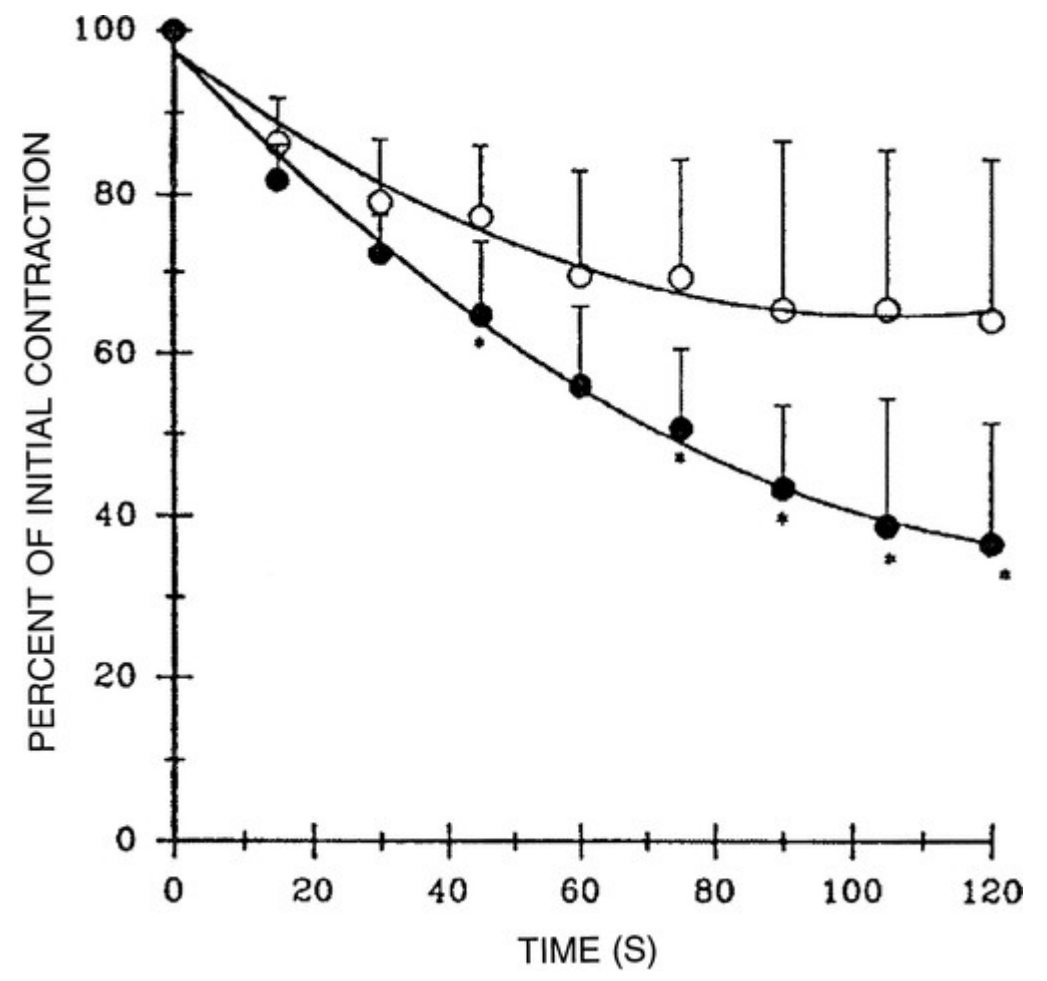

Fig. 7. Isometric fatigue data of control (o) and flight $(\bullet)$ soleus muscles. Flight muscles were more fatigable than control muscles. Data points for both groups of muscles were fitted by using a second-order polynomial. Values are means \pm SD. $* P<0.05$. [From Caiozzo et al. (11).]

Similar to the flight condition, we have previously shown $\mathrm{HU}$ to increase the fatigability of the Sol (61). The increased fatigue could not be attributed to an altered steady-state blood flow (61). Recently, our laboratory found that $2 \mathrm{wk}$ of $\mathrm{HU}$ had no effect on the ability of homogenate or mitochondrial fractions of the Sol, red gastrocnemius, or white gastrocnemius to oxidize either pyruvate or palmitate (36). However, the increased fatigue in the Sol of the HU animal was associated with a greater rate of glycogen depletion and lactate production in the type I fibers compared with the control group. The failure to observe any significant decline in palmitate oxidation suggests that the enzymes required for 
fat oxidation were not altered. One hypothesis consistent with the data is that glycolysis is activated and fatty acid oxidation inhibited by substrate-level control. Although $\mathrm{HU}$ in rats (and perhaps SF) does not affect steady-state blood flow during exercise, it has been shown to reduce the endotheliumdependent dilation in Sol feed arteries (45). Thus the possibility exists that models of weightlessness and SF may reduce the rate at which muscle blood flow increases with the onset of exercise. If true, the decrease in creatine phosphate and ATP, and the increase in ADP, AMP, and $P_{i}$, would be greater in the muscles of the unloaded subjects. The high AMP and $\mathrm{P}_{\mathrm{i}}$ would stimulate glycolysis and the production of acetyl-CoA. The latter would act as a substrate for malonyl-CoA, thus reducing the exercise-induced decline in this substrate (101). Because malonyl-CoA is a known inhibitor of CPT I, fatty acid oxidation would be reduced. The increased fatigue could be explained by the elevated Piand $\mathrm{H}^{+}$, which are both known to directly inhibit cross-bridge force production (28).

\section{SF AND MUSCLE FIBER DAMAGE}

\section{Relationship of inflight changes to muscle cell damage.}

Humans returning to Earth after SF frequently experienced muscle weakness and delayed-onset muscle soreness conditions indicative of muscle cell damage (1ㅗ 102). However, until the 1993 SLS-2 mission, during which tissues were harvested in space, it was impossible to separate inflight from postflight changes in cell structure (9). The SLS-2 studies demonstrated that, at least in rats, fiber lesions were not observed inflight in either the Sol or AL muscles. This experiment proved what had previously been suspected: muscle fiber damage was primarily a postflight phenomena, likely resulting from eccentric contractions during reloading in a $1-G$ environment $(\underline{77}, \underline{80}, \underline{81})$.

Although SF does not usually induce muscle cell damage, it establishes a condition within the fibers that increases their susceptibility for damage during postflight reloading. The question that remains to be answered is, What are these factors? It is well established that reloading after either SF or $\mathrm{HU}$ in rats causes selective fiber damage in the slow type I fiber type (1ㅣ). When the entire AL was maximally activated by electrical stimulation post-HU, the fast-twitch type II fibers showed the greatest susceptibility to damage (unpublished observations). Thus the selective damage of type I fibers during normal reloading activities such as walking may simply reflect the preferential recruitment of this fiber type. Any load would be relatively greater on the atrophied fiber and thus more likely to cause damage postflight. In addition to the increased relative load and selective recruitment, SF may induce qualitative changes in cell proteins in the type I fiber that leads to a more fragile fiber with reloading. For example, SF has been shown to cause a selective loss of the actin thin filaments over the myosin thick filaments in the slow type I fiber (Ref. 76; Widrick, Knuth, Norenberg, Romatowski, Bain, Riley, Karhanek, Trappe, Trappe, Costill, and Fitts, unpublished observations). Although the atrophied fiber had a reduced absolute tension, its specific tension was near normal. We interpreted this to mean that the normal thick-to-thin filament ratio of 6:1 supplies an excess of actin-binding sites for myosin cross bridges and that reducing the ratio to $\sim 5: 1$ during SF continues to provide sufficient cross-bridge binding to maintain a near-normal specific tension (Widrick, Knuth, Norenberg, Romatowski, Bain, Riley, Karhanek, Trappe, Trappe, Costill, and Fitts, unpublished observations). However, during postflight eccentric contractions, the average stress per remaining thin filament would significantly increase, which might increase the susceptibility of the atrophic muscles to reloading damage. A second possibility would be a selective loss in linking proteins that normally transmit contractile force 
to the extracellular matrix. For example, reduction or absence of a single component of the dystrophinglycoprotein complex can result in greater susceptibility to contraction-induced tearing of the sarcolemma. Currently, it is unknown whether SF alters this complex. The data reviewed here suggest that most fiber damage occurs during postflight reloading. However, extravehicular activity (space walks) during the Hubble telescope repair mission was reported to produce muscle soreness. This suggests that some fiber damage in humans might occur inflight. This question cannot be answered until inflight biopsies are made on the ISS.

Postflight muscle fiber damage.

The SLS-2 mission documented for the first time that at least in rats muscle fiber damage did not occur inflight (79). At $3 \mathrm{~h}$ postflight, no lesions were detected in the Sol or AL, whereas by $4.5 \mathrm{~h}$ eccentric contraction-like sarcomere disruptions were detected in $2.8 \%$ (SLS-1) and $2.4 \%$ (SLS-2) of the myofibers in the caudal slow region of the $A L(\underline{79})$. The lesions were significantly more prevalent in the $A L$ myofibers of the caudal slow region than the rostral mixed fast and slow region, an observation consistent with slow fibers being more susceptible to damage. No lesions were observed in the Sol at anytime postflight, and by 9 days no lesions remained in the $A L$, indicating that sarcomere repair was complete. The postflight posture of the animals seemed to explain the selective damage of the AL (79)). The postflight rats stood erect less frequently on the hindlegs, and, when standing, weight bearing was on the heels without normal plantar flexion. Thus Sol contractile activity was reduced relative to the control rats. When standing, the postflight rats were postured closer to the ground, which produced greater flexion of the hip joints. Biomechanically, this caused the AL muscles, which are hip adductors and extensors, to be stretched and bear weight.

The earlier Cosmos flights provided important data on the time course of the lesion development. It was clear from the SLS-2 results that postflight fiber lesions developed within hours of landing, whereas the Cosmos 1887 12.5-day flight, in which rats were killed 2 days after landing, showed that extensive muscle fiber necrosis, activated macrophages, and satellite cells, and interstitial edema were present ( extensive sarcomere disruption and edema but minimal tissue necrosis, macrophage, and satellite cell activation (77). The postflight fiber lesions appear to be primarily induced by the degree of strain and the level of force. The high degree of lesions in the Sol (15\%) and AL (44\%) muscles after the Cosmos 2044 mission may have been due in part to the higher $\mathrm{G}$ force of parachute landing compared with the SLS-1 and SLS-2 shuttle landing on a runway. The fiber lesions postflight do not appear to have been mediated by $\mathrm{Ca}^{2+}$-activiated proteases, but ubiquitin-mediated proteolysis may play a role (그).

\section{EXERCISE COUNTERMEASURES}

\section{Animal studies.}

The rat HU model has been used extensively to assess the effectiveness of various exercise countermeasures (21). Edgerton and Roy (21) recently reviewed this topic in detail, and thus only an overview of important features will be presented here. Edgerton and colleagues $(\underline{40}, \underline{42}, \underline{70})$, Booth and colleagues $(\underline{18})$, and Fitts and colleagues $(\underline{9}, \underline{96}, \underline{100})$ have all provided convincing evidence that in rats short periods of exercise interspersed throughout the day were more effective than one long daily bout. The speculation was that intermittent bouts of exercise were more effective in maintaining 
protein synthesis at control levels during the unweighting period (18). For example, during a 7-day HU period, 10 -min bouts of standing or slow walking on a treadmill repeated four times daily maintained a near-normal soleus mass $(\underline{18}, \underline{42})$. The data of Widrick et al. $(\underline{96})$ suggest that this is particularly true for short periods of unweighting of up to 7 days. When $\mathrm{HU}$ was extended to 14 days, four 10 -min periods of standing per day reduced the loss of soleus-to-body weight ratio by $22 \%$ and attenuated alterations in type I fiber diameter and peak force by 36 and 29\%, respectively. Clearly a simple, intermittentstanding protocol was less effective over 14 days compared with 7 days of HU. In rats, the first week of muscle wasting with $\mathrm{HU}$ is primarily caused by a decline in protein synthesis, whereas myofibril degradation does not reach its maximum until days 9-15 (2) $)$. Perhaps the intermittent-standing protocol was more effective in preventing the decline in protein synthesis than it was in reducing degradation.

The only exercise paradigm known to induce fiber hypertrophy is high-resistance weight training (27). Despite this, very little data exist on the effectiveness of this exercise modality in the prevention of atrophy incurred by models of unloading or SF. The best controlled and quantified data come from rat $\mathrm{HU}$ studies. Ten minutes of standing with high-resistance intermittent exercise (ten $15-\mathrm{cm}$ climbs with 500-g weights, $1 \mathrm{climb} / \mathrm{min}$ ) four times per day was more effective in preventing fiber atrophy than standing alone (97). Kirby et al. (52) employed electrical stimulation to elicit four sets of six repetitions of eccentric contractions every other day during a 10-day HU period. This paradigm reduced the loss of muscle mass and noncollageneous proteins by 77 and $44 \%$, respectively. In another high-resistance exercise study, Grindeland et al. (38) reported that daily administration of human GH plus ladder climbing prevented Sol muscle atrophy in 10-day HU rats. The combined hormone plus exercise paradigm was more effective than either treatment alone. Collectively, these data suggest that effective exercise countermeasure programs will need to be not only frequent but also contain a component of high-resistance exercise. How much and what type of high-resistance exercise remain to be defined. For example, we have preliminary data that suggest that two 5-s isometric contractions performed three times per day is sufficient to maintain Sol muscle mass during a 14-day period of HU in rats (unpublished observations). However, to protect both slow and fast muscles, the optimal exercise countermeasure might require high-resistance exercise that utilizes both isometric and isotonic contractions. Additionally, eccentric contractions have been shown to reduce muscle atrophy, but they also increase the susceptibility to fiber damage. Thus any program employing eccentric contractions needs to be carefully designed to maximize the anabolic affects while preventing fiber damage.

\section{Human studies.}

Exercise countermeasures have been used extensively during both long-duration (Skylab and Mir) and short-duration (Spacelab) SF $(\underline{15}, \underline{35})$. For the most part, the exercise has consisted of aerobic activities using cycle ergometers and treadmills. It has been difficult to determine the effectiveness of these programs because nonexercising controls have not been studied, and the actual exercise protocols have generally not been well described. For example, during the Skylab missions, the only quantitative data on inflight exercise were obtained from the cycle ergometer. The mean workload was highest in mission 4, and, despite being the longest Skylab mission, leg strength showed the least decline. However, the improved performance could not be attributed to a given exercise paradigm because the crew exercised on more devices than utilized by the earlier Skylab 2 and 3 crews (프). 
Perhaps the best data on the benefits of exercise countermeasures in humans have come from bedrest studies $(\underline{8}, \underline{34})$. The primary findings from these studies is that high-resistance exercise training is effective in preventing muscle wasting and reduced performance. Furthermore, it appears that humans may require less frequent bouts of exercise to maintain muscle function. Bamman et al. (으) found that five sets of 6-10 repetitions to failure of constant-resistance concentric and eccentric plantar flexion performed every other day during 14 days of bed rest was enough to prevent any drop in torque or power at all velocities tested. One concern is the specificity of the exercise employed. Greenleaf et al. (34) demonstrated that isokinetic knee flexion-extension exercise during a 30-day bedrest study actually increased knee extension total work by $27 \%$ compared with a $16 \%$ decrease with nonexercising bed-rest subjects. A group performing high-intensity lower extremity cycle ergometry maintained a total work capacity similar to their pre-bed-rest values. The knee testing was conducted on the same device as the isokinetic knee flexion-extension exercise countermeasure, and the greater benefits of this exercise training likely reflected the specificity of training. A recent publication by Bamman and Caruso (의) perhaps best demonstrates the specificity of training. During a 14-day bed rest, seven subjects performed five sets of leg press exercise to volitional fatigue (6-10 repetitions) every other day. Before and immediately after the bed rest, the nonexercising and exercising bed-rest subjects were tested by using an isokinetic concentric-eccentric knee-extension protocol. Anglespecific torque and power fell by 18 and $20 \%$, respectively, and there were no differences between the nonexercising and exercising groups. The specificity of training was demonstrated by the observation that the resistance training protocol had previously been shown to prevent muscle fiber atrophy and strength loss during a one-repetition-maximum constant load lift (ㄱ). The authors suggest that most movements on Earth require constant external loaded contractions, and thus inflight exercise countermeasures should emphasize constant external loading rather than isokinetic techniques (으).

Clearly, studies on the ISS will be needed to sort out the most appropriate exercise countermeasure in humans. Rat data suggest that the "ideal program" should include isometric as well as isotonic (concentric and eccentric) exercise, and both human and rat data indicate high-intensity exercise as the modality of choice for the protection of limb muscle structure and function.

\section{CONCLUSIONS AND FUTURE DIRECTIONS}

\section{Conclusions.}

A consistent feature of SF regardless of the species is limb muscle atrophy and the loss of peak force and power. Species differences exist in the rate and mechanisms of muscle wasting and in the susceptibility of a given fiber type to atrophy. Rat skeletal muscle shows preferential type I fiber atrophy and faster wasting than nonhuman primate and human muscle. In contrast, for humans, the type II fiber shows equal or greater atrophy in response to microgravity than the type I fiber. The faster muscle wasting in rats might be in part due to the SF-induced decrease in protein synthesis coupled with an increased degradation, whereas in human muscle only the former appears to occur. In both rats and humans, the extensor muscles are the first to be affected by microgravity, but Mir experiments suggest that by 6 mo in humans both extensors and flexor show similar levels of atrophy. The calf muscle is one of the most affected limb muscles, and in all species the Sol shows more microgravity-induced atrophy than the gastrocnemius. The increased susceptibility of the Sol to microgravity likely results from both the removal of the antigravity load as well as a shift in the 
neuronal recruitment pattern from primarily Sol activation to a greater involvement of the gastrocnemius.

Besides the quantitative change in muscle mass, SF induces important qualitative changes in the structure of skeletal muscle. It is clear that contractile proteins are lost out of proportion to other cellular proteins and that the actin thin filament is lost disproportionately to the myosin thick filament. The former explains the decrease in force per CSA, whereas the latter has been hypothesized to be responsible for the $\sim 30 \%$ postflight increase in type I fiber $V_{0}$. This adaptation allows the muscle to partially protect against the decline in peak power caused by the reduced force, by shortening at higher velocities. The increased filament spacing could also explain the greater drop in fiber stiffness relative to force and the small right shift in the type I fiber force-pCa relationship that is observed in some fibers postflight.

Molecular studies indicate that the reduced protein synthesis induced by unloading can be attributed to both a downregulation of gene expression and to a reduced rate of translation. Postflight, 10-15\% of slow fibers have been shown to coexpress fast-type myosin isozymes. In a small percentage of fibers, a complete shift appears to occur such that postflight there is an increased number of fast type II fibers. At least for myosin, the increased fast isoform expression appears to be caused by activation of regulatory sequences within the fast MHC promoter. The increased number of hybrid and fast fibers would contribute to the increased velocity measured in whole muscles after SF. The shortened isometric twitch duration in the rat Sol after $\mathrm{HU}$ can be attributed in part to a large increase in fasttype SR protein; it is not known whether SF induces a similar change. The increased occurrence of type I fiber damage postflight may also have its etiology in the structural rearrangement of the myofilaments. Clearly, any load would be relatively greater on the atrophied fiber and thus more likely to cause damage postflight. Additionally, postflight, the average stress per remaining thin filament would significantly increase, which might increase the susceptibility to fiber damage.

Although mitochondrial proteins are lost in response to SF, the decline is less than that observed for contractile proteins. As a result, the mitochondrial protein content (mg protein/g fiber) either is unaltered or shows a slight increase after SF. Despite this, an increased fatigability has been reported by astronauts, and postflight isolated rat Sol muscles fatigue more rapidly. Associated with the increased fatigue is a reduced ability of homogenates from postflight rat skeletal muscle to oxidize palmitate. The cause of the increased fatigue and reduced fat oxidation is unknown. One possibility is that postflight exercise elicits a greater activation of glycolysis and increase in $\mathrm{H}^{+}$and $\mathrm{P}_{\mathrm{i}}$.

\section{Future directions.}

Research over the past 30 years has made important progress toward understanding the effects of SF on skeletal muscle. Alterations with short-term SF have been extensively studied, and the extent of muscle atrophy and loss of functional capacity resulting from 1 to $3 \mathrm{wk}$ of microgravity is well established. Future ISS studies are needed to define the effects of long-term SF such that the time course of muscle atrophy and the steady-state functional change with microgravity can be established. With this knowledge, we will be able to predict the changes in muscle structure and function that can be expected in a mission to Mars and back. 
An important future goal will be to develop effective exercise countermeasures to prevent or limit muscle atrophy and functional losses in force and power. To achieve this goal, it will be important to elucidate the cellular and molecular mechanisms responsible for muscle cell wasting. To establish fiber type-specific effects, molecular and cellular studies will need to be carried out on individual fibers. With this mechanistic information, it should be easier to develop effective countermeasures. Research on this subject suggests that the most effective exercise countermeasure will likely employ highresistance exercise performed in short but frequent bouts throughout the day. However, this information comes primarily from $\mathrm{HU}$ rat studies. In the future, it will be important to conduct wellcontrolled exercise countermeasure studies in humans by using the bed-rest model and ultimately the ISS. The goal of these studies should be to establish the optimal mixture of isometric and isotonic (concentric and eccentric) exercise and the frequency of bouts required to prevent microgravityinduced muscle atrophy. Additionally, these studies should address the cause of the individual differences known to exist in microgravity-induced muscle atrophy. Does this variability have a molecular cause or simply result from different degrees of fitness and exercise countermeasures?

Human studies using bed rest and the ISS need to be designed to determine whether substrate utilization is altered as a result of unloading. Is there an increase in glycogen utilization and lactate production, and can the increased fatigue postflight be explained by altered patterns of fuel utilization? Finally, it will be important to understand the mechanisms responsible for the increased susceptibility of muscle damage on return to the $1-G$ environment and to establish that the exercise countermeasures protect against this problem.

R. H. Fitts thanks his current research assistant Janell Romatowski and his former graduate students and postdoctoral fellows who contributed to the space biology research effort of the laboratory over the past 25 years.

\section{FOOTNOTES}

- This work was supported in part by National Aeronautics and Space Administration Grants NAS 9-18768 and NAG 9-1156 (to R. H. Fitts).

- Address for reprint requests and other correspondence: R. H. Fitts, Marquette Univ., Dept. of Biology, Wehr Life Sciences Bldg., P.O. Box 1881, Milwaukee, WI 53201-1881 (Email:robert.fitts@marquette.edu).

- The costs of publication of this article were defrayed in part by the payment of page charges. The article must therefore be hereby marked "advertisement" in accordance with 18 U.S.C. $\S 1734$ solely to indicate this fact.

\section{We recommend}

1. Altered actin and myosin expression in muscle during exposure to microgravity

D. B. Thomason et al., Journal of Applied Physiology, 1992

\section{Elevated interstitial fluid volume in rat soleus muscles by hindlimb unweighting}

S. C. Kandarian et al., Journal of Applied Physiology, 1991 
3. Reactive oxygen species upregulate expression of muscle atrophy-associated ubiquitin ligase Cbl-b in rat L6 skeletal muscle cells

Takayuki Uchida et al., American Journal of Physiology - Cell Physiology, 2018

4. Skeletal muscle atrophy in response to 14 days of weightlessness: vastus medialis

X. J. Musacchia et al., Journal of Applied Physiology, 1992

5. Skeletal muscle adaptations to microgravity exposure in the mouse

B. C. Harrison et al., Journal of Applied Physiology,2003

1. Chemotherapy Plays a Role in Skeletal Muscle Atrophy in Patients With Breast Cancer

Leah Lawrence, Cancer Therapy Advisor, 2018

2. Invitae, Biogen Partner on Free Spinal Muscular Atrophy Genetic Testing Program

360Dx, 2018

3. Fish Oil Supplementation Can Mitigate Disuse Atrophy in Women

Medscape

4. Role of Protein Carbonylation in Skeletal Muscle Mass Loss Associated with Chronic Conditions

Barreiro, Esther et al., Proteomes, 2016

5. Overexpression of Glucocorticoid Receptor $\beta$ Enhances Myogenesis and Reduces Catabolic Gene Expression

Hinds, Terry D. et al., Int J Mol Sci, 2016 